\title{
Curcumin upregulates Nrf2 nuclear translocation and protects rat hepatic stellate cells against oxidative stress
}

\author{
ZHENXIONG LIU $^{1 *}$, WEIJIA DOU ${ }^{1 *}$, YUANYUAN ZHENG ${ }^{2 *}$, QINSHENG WEN $^{1}$, MING QIN $^{1}$, XUXIA WANG $^{1}$, \\ HUA TANG $^{1}$, RONG ZHANG ${ }^{1}$, DANDAN LV ${ }^{1}$, JINGJIE WANG ${ }^{1}$ and SHUGUANG ZHAO ${ }^{1}$ \\ ${ }^{1}$ Department of Gastroenterology, Tangdu Hospital, Fourth Military Medical University, Xi'an, Shaanxi 710038; \\ ${ }^{2}$ Department of Gastroenterology, 180 Military Hospital, Quanzhou, Fujian 362000, P.R. China
}

Received January 25, 2015; Accepted November 25, 2015

DOI: $10.3892 / \mathrm{mmr} .2015 .4690$

\begin{abstract}
The present study aimed to investigate the protective role of curcumin against oxidative stress in rat hepatic stellate cells (HSCs)-T6, and to determine the possible underlying mechanisms. HSC-T6 cells were divided into three groups: Negative control group, oxidant-treated group and curcumin-treated group. Flow cytometry and spectrophotometry were used to measure the production of reactive oxygen species (ROS), and the levels of malondialdehyde (MDA) and glutathione (GSH). Immunocytochemistry and a radioimmunoassay were used to determine the expression of smooth muscle $\alpha$-actin ( $\alpha$-SMA) and the secretion of extracellular matrix (ECM) molecules. In addition, western blotting and immunocytochemistry were used to determine the expression levels of nuclear factor-erythroid 2-related factor (Nrf2). Treatment with glucose oxidase (GO) significantly stimulated the formation of ROS and increased the production of MDA, as compared with the control cells; however, the production of GSH was only slightly increased. In addition, treatment with GO significantly promoted the expression of $\alpha$-SMA and the secretion of ECM molecules. Conversely, treatment with curcumin significantly decreased the levels of ROS and MDA, and significantly increased the
\end{abstract}

Correspondence to: Professor Jingjie Wang or Professor Shuguang Zhao, Department of Gastroenterology, Tangdu Hospital, Fourth Military Medical University, 569 Xinsi Road, Xi'an, Shaanxi 710038, P.R. China

E-mail: jingjie@fmmu.edu.cn

E-mail: zsg1203@126.com

*Contributed equally

Abbreviations: Nrf2, nuclear factor-erythroid 2-related factor; HSC, hepatic stellate cell; GO, glucose oxidase; ROS, reactive oxygen species; MDA, malondialdehyde; GSH, glutathione; $\alpha$-SMA, smooth muscle $\alpha$-actin; ECM, extracellular matrix; PCIII, type III procollagen; C IV, type IV collagen; LN, laminin; HA, hyaluronic acid; DHE, dihydroethidium

Key words: curcumin, nuclear factor-erythroid 2-related factor, oxidative stress, hepatic stellate cell, fibrosis levels of GSH. Curcumin significantly inhibited the expression of $\alpha$-SMA and decreased the secretion of ECM molecules. Furthermore, treatment with curcumin significantly increased the nuclear expression levels of Nrf2. These results indicated that curcumin may protect rat HSCs against oxidative stress and inhibit the GO-induced activation and secretion of ECM molecules in vitro. These effects were mediated by the upregulation of Nrf2 nuclear translocation.

\section{Introduction}

Previous studies have demonstrated that oxidative stress has a significant role in the occurrence and progression of hepatitis and hepatic fibrosis (1-4). Oxidative stress results from the excessive production of reactive oxygen species (ROS), and the inability of an organism to eliminate them. Excessive ROS lead to lipid peroxidation, protein and DNA damage, and injury to cellular structure and function (5). Numerous studies have reported the important role of ROS in various types of hepatic injury (6-10). In addition to ROS-induced inflammation, ROS may lead to loss of normal regulatory functions, resulting in tissue injury and excessive repair, and the development of hepatitis and hepatic fibrosis $(11,12)$. It has previously been demonstrated that activation of hepatic stellate cells (HSCs) has a key role in the progression of hepatic fibrosis; therefore, HSCs are considered important target cells in hepatic fibrosis research (13). Furthermore, HSCs can be activated by oxidative stress and transformed into myofibroblasts. Myofibroblasts synthesize abundant extracellular matrix (ECM) molecules, which may lead to hepatic fibrosis (14); therefore, how to inhibit the activation of HSCs exposed to oxidative stress requires further investigation. Nuclear factor-erythroid 2-related factor (Nrf2) is a transcription factor that activates numerous antioxidant enzymes and phase II detoxifying enzymes (15). In addition, Nrf2 has an important role regulating oxidative stress $(16,17)$; however, the majority of studies regarding Nrf2 have focused on the nervous and respiratory systems. The effects of Nrf2 on HSCs, and the underlying molecular mechanisms, have seldom been reported.

The present study hypothesized that upregulation of Nrf2 nuclear translocation would promote the expression of antioxidant enzymes and phase II detoxifying enzymes, thus protecting the liver against injury. The present study investigated the 
alterations and regulatory mechanisms of the Nrf2 pathway, which is of great significance for understanding the pathogenesis of hepatic fibrosis and developing novel preventative strategies and curative therapies.

Curcumin, which is an ingredient of the spice turmeric, is present in the rhizomes of Curcuma longa Linn (Zingiberaceae). Curcumin has been reported to exert antioxidant, anti-inflammatory, anticancer and hepatoprotective effects (18). Furthermore, curcumin functions as an exogenous Nrf2 agonist, and can promote the nuclear translocation and biological effects of Nrf2 (19). Therefore, the present study used curcumin to upregulate Nrf2, and subsequently investigated the effects of $\mathrm{Nrf} 2$ on HSCs.

To investigate the possible regulatory mechanisms that underlie the Nrf2 pathway, the present study examined the effects of curcumin on Nrf2. In addition, alterations in the levels of ROS, malondialdehyde (MDA) and glutathione (GSH) were detected. As an index of HSC activation, smooth muscle $\alpha$-actin ( $\alpha$-SMA) and desmin levels were measured. Furthermore, ECM-secreted proteins, including type III procollagen (PCIII), type IV collagen (CIV), laminin (LN) and hyaluronic acid (HA), were measured following treatment of HSCs with curcumin. The results of the present study indicated that curcumin was able to protect HSCs against oxidative stress, and inhibit the activation of HSCs via induction of Nrf2 nuclear translocation.

\section{Materials and methods}

Materials. Curcumin and GO were purchased from Sigma-Aldrich (St. Louis, MO, USA). Anti- $\alpha$-SMA (1:200; cat. no. sc-53142) and anti- $\beta$-actin (1:500; cat. no. sc-47778) antibodies were obtained from Santa Cruz Biotechnology, Inc. (Dallas, TX, USA). Anti-Nrf2 (1:500; cat. no. BS6286) and anti-desmin (1:500; cat. no. BS1712) antibodies were purchased from Bioworld Technology, Inc. (St. Louis Park, MN, USA). Horseradish peroxidase-conjugated anti-rabbit (1:10,000; cat. no. bs-0295G-HRP) and anti-mouse (1:10,000; cat. no. bs-0296G-HRP) immunoglobulin (Ig)G, and fluorescein isothiocyanate (FITC)-conjugated anti-rabbit (1:1,000; cat. no. bs-0295G-FITC) and anti-mouse (1:1,000; cat. no. bs-0296G-FITC) IgG secondary antibodies were obtained from Beijing Biosynthesis Biotechnology Co., Ltd. (Beijing, China). Dihydroethidium (DHE) was purchased from Beyotime Institute of Biotechnology (Jiangsu, China). MDA and GSH kits were obtained from Nanjing Jiancheng Biotechnology, Inc. (Nanjing, China). PCIII, CIV, LN and HA kits were purchased from the Shanghai Naval Medical Institute (Shanghai, China).

Cell culture. The HSC-T6 immortalized rat HSC line exhibits a stable phenotype and biochemical characteristics (20). HSC-T6 cells were a generous gift from Dr Ding of the Medical College of Xi'an Jiaotong University (Xi'an, China). The cells were grown under standard conditions in a normoxic atmosphere in high-glucose Dulbecco's modified Eagle's medium (Gibco; Thermo Fisher Scientific, Inc., Waltham, MA, USA) supplemented with $10 \%$ fetal bovine serum (FBS; Sijiqing, Hangzhou, China) in a humidified incubator containing $5 \% \mathrm{CO}_{2}$ at $37^{\circ} \mathrm{C}$. All subsequent experiments were conducted using cells at the exponential stage of growth. Cells were seeded into a $25 \mathrm{~cm}^{2}$ plastic culture flask at a density of $1 \times 10^{6}$ cells. The cells were separated into three groups: The negative control cells were incubated with $5 \mathrm{ml}$ culture medium; the oxidant-treated cells were incubated with $5 \mathrm{ml}$ culture medium supplemented with $100 \mathrm{mU} / \mathrm{ml}$ GO for $2 \mathrm{~h}$ before each experimental manipulation; and the curcumin-treated cells were pre-treated with $5 \mathrm{ml}$ culture medium containing $0.15 \mu \mathrm{mol}$ curcumin for $3 \mathrm{~h}$, and then incubated in the same manner as the oxidant-treated cells.

Western blot analysis. Total, cytoplasmic and nuclear proteins were obtained from the cells using protein extraction kits (Beyotime Institute of Biotechnology, Shanghai, China), according to the manufacturer's protocol. The concentration of the protein samples was quantified using a Bradford Protein Assay kit (Beyotime Institute of Biotechnology), according to the manufacturer's protocol. Subsequently, the protein samples $(15 \mu \mathrm{g})$ were separated by $10 \%$ sodium dodecyl sulfate-polyacrylamide gel electrophoresis and were transferred to nitrocellulose membranes (Bio-Rad Laboratories, Inc., Hercules, CA, USA). The membranes were blocked in buffer containing skim milk for $2 \mathrm{~h}$, and were then incubated with anti-Nrf2 primary antibody at $4^{\circ} \mathrm{C}$ overnight, and washed with phosphate-buffered saline (PBS). The membranes were then incubated with a horseradish peroxidase-conjugated secondary antibody at room temperature for $1 \mathrm{~h}$. Pierce Enhanced Chemiluminescence Western Blotting substrate (Thermo Fisher Scientific, Inc.) was used to develop the blots, and immunoreactivity was visualized following exposure to X-OMAT BT Film (Beyotime Institute of Biotechnology). $\beta$-actin was used as an internal control. The results were quantified and normalized to $\beta$-actin using Molecular Analyst software (version 1.4.1; Bio-Rad Laboratories, Inc.)

Immunocytochemistry. Following a $24 \mathrm{~h}$ cell culture, in which the cells became adherent, immunocytochemistry was conducted. Cell-coated dishes $\left(1 \times 10^{5}\right.$ cells $\left./ \mathrm{ml}\right)$ were fixed with $4 \%$ paraformaldehyde for $30 \mathrm{~min}$ and washed with PBS. The cells were permeabilized using 0.3\% Triton X-100 (Sigma-Aldrich) for $15 \mathrm{~min}$. Endogenous peroxidases and biotins were quenched using $3 \% \mathrm{H}_{2} \mathrm{O}_{2}$. The cells were then blocked with FBS and incubated with the indicated primary antibodies at $4^{\circ} \mathrm{C}$ overnight. Sections stained for Nrf2 protein expression were washed and incubated with a horseradish peroxidase-conjugated secondary antibody at room temperature for $30 \mathrm{~min}$. Bound secondary antibodies were detected using Histostain-SP kits (ZSbio, Beijing, China), according to the manufacturer's protocol. The reaction products were visualized using diaminobenzidine tetrahydro-chloride (Tiangen Biotech Co., Ltd., Beijing, China). Sections stained for $\alpha$-SMA and desmin protein expression were incubated with FITC-conjugated secondary antibodies at room temperature for $30 \mathrm{~min}$ in the dark. The nuclei were stained using Evans Blue (Sigma-Aldrich) in sections stained for $\alpha$-SMA. In the negative control group, primary antibodies were substituted with PBS. Stained sections were viewed under a Nikon Eclipse 800 fluorescent microscope (Nikon Corporation, Tokyo, Japan).

Flow cytometry. HSC-T6 cells were maintained under standard conditions, and were then transferred to culture dishes. Following a $24 \mathrm{~h}$ culture, $1 \times 10^{6}$ cells from each group were 
placed in tubes. The cells were washed with PBS and separated by centrifugation at $432 \mathrm{x} \mathrm{g}$ for $10 \mathrm{~min}$ at $4^{\circ} \mathrm{C}$. Serum-free media supplemented with $2-\mu \mathrm{M}$ DHE were added, and the tubes were incubated at $4^{\circ} \mathrm{C}$ for $30 \mathrm{~min}$ in the dark. The remaining cells were washed with PBS and subjected to further centrifugation at $432 \mathrm{x} \mathrm{g}$ for $10 \mathrm{~min}$ at $4^{\circ} \mathrm{C}$. Paraformaldehyde $(2 \mathrm{ml} ; 4 \%)$ was added to each tube and the tubes were incubated at $4^{\circ} \mathrm{C}$ for $30 \mathrm{~min}$ in the dark, followed by centrifugation at $432 \mathrm{x} \mathrm{g}$ for $10 \mathrm{~min}$ at $4^{\circ} \mathrm{C}$. Subsequently, $300 \mu \mathrm{l}$ PBS was added to the preparations and mixed gently. DHE fluorescence was measured by flow cytometry (FACSCanto II; BD Biosciences, Franklin Lakes, NJ, USA).

MDA and GSH assays.HSC-T6s were incubated in culture dishes and the supernatants were collected for the detection of MDA and GSH by spectrophotometry. Measurement of the product (MDA-TBA adduct) of a reaction between MDA and 2-thiobarbituric acid, and of the product (2-nitro-5-sulphur benzoic acid) of a reaction between GSH and dithio-dinitrobenzoic acid allow the levels of MDA and GSH to be analyzed using colorimetric assays. MDA and GSH levels were determined using kits, according to the manufacturers' protocols. Absorbance was measured at 532 and $412 \mathrm{~nm}$ using a spectrophotometer (UV-2450; Shimadzu Corporation, Kyoto, Japan) for MDA and GSH, respectively. The concentrations of MDA and GSH were calculated according to the equation provided in the kits.

Analysis of ECM secretion. Cells were initally separated into three groups and cultured in serum-free medium overnight. Subsequently, the cells were treated as mentioned previously (negative control, oxidant-treated and curcumin-treated cells). Following centrifugation at $1,000 \times \mathrm{g}$ for $20 \mathrm{~min}$ at $4^{\circ} \mathrm{C}$, the supernatant was collected and maintained at $-80^{\circ} \mathrm{C}$ until further analysis. The levels of PCIII, CIV, LN and HA secreted into the supernatant were analyzed using commercially available radioimmunoassay kits, according to the manufacturers' protocols.

Statistical analysis. The data are presented as the mean \pm standard deviation and significance was assessed using SPSS 12.0 software (SPSS, Inc., Chicago, IL, USA). Statistical comparisons were performed using one-way analysis of variance. Paired comparisons were conducted using Student Newman Keuls-q test. $\mathrm{P}<0.05$ was considered to indicate a statistically significant difference.

\section{Results}

Curcumin protects $\mathrm{HSCs}$ against $\mathrm{GO}$-induced oxidative stress injury. As a marker of oxidative stress, GO may react with glucose in the culture media and subsequently promote the generation of glucuronic acid and hydrogen peroxide $\left(\mathrm{H}_{2} \mathrm{O}_{2}\right)$. It is well known that DHE, which is a superoxide radical-specific fluorescent probe, is able to enter viable cells freely where it is oxidized by superoxide to form ethidium, which binds to DNA and exhibits red fluorescence. Therefore, the present study detected fluorescence intensity in order to estimate the relative levels of ROS production. As shown in Fig. 1A, compared with the negative control cells, treatment of the cells with GO significantly increased fluorescence intensity, whereas pretreatment with curcumin significantly decreased fluorescence intensity.
However, the fluorescence intensity in the curcumin-treated cells was stronger, as compared with in the negative control cells.

Furthermore, ROS may react with lipids in cell and mitochondrial membranes, resulting in the production of MDA, which can be used to measure lipid peroxidation. Due to its strong cytotoxicity, MDA is able to alter membrane permeability or disrupt membrane integrity, leading to oxidative injury. However, even in the case of increased lipid peroxidation, oxidative stress only occurs in cells that are unable to defend and protect against free radical injury or chemically induced damage. GSH is an important endogenous antioxidant, which reduces levels of ROS. GSH acts as a free radical scavenger, a coenzyme for various antioxidant enzymes, a regulator of thiol-disulfide status, and is involved in the detoxification of electrophilic xenobiotics via conjugation. Therefore, the present study aimed to detect the levels of MDA and GSH in the supernatant. Significantly enhanced levels of MDA were detected in the oxidant-treated cells, as compared with the negative control cells, which were correlated with the levels of ROS. The MDA levels were markedly attenuated in response to curcumin pretreatment, however they remained higher than in the control cells. Furthermore, compared with the negative control cells, the levels of GSH were slightly increased in the oxidant-treated cells, whereas they were significantly elevated in the curcumin-treated cells (Fig. 1B).

These results suggest that an oxidative stress model of HSC-T6 was established with GO treatment, as demonstrated in the increased levels of ROS and MDA. Furthermore, pretreatment with curcumin was able to significantly suppress the degree of oxidative stress, at least partially due to the induced expression of endogenous GSH.

Curcumin promotes the nuclear translocation of $\mathrm{Nrf} 2$. GSH synthesis is governed by Nrf2 via the regulation of the rate-limiting enzymes glutamate cysteine ligase (GCL) catalytic subunit (GCLC) and GCL modifier subunit (GCLM). To elucidate the molecular mechanism by which curcumin protects HSCs against oxidative stress injury, the present study detected the expression of Nrf2 in HSC-T6 cells by immunocytochemistry (Fig. 2A) and western blot analysis (Fig. 2B and C). As expected, the expression of total Nrf2 did not differ between the groups; however, the expression levels of cytoplasmic Nrf2 were slightly decreased in the oxidant-treated cells, and significantly decreased in the curcumin-treated cells, as compared with the negative control cells. Conversely, the expression of nuclear Nrf2 was absent in the negative control cells and was only slightly expressed in the oxidant-treated cells; however, the expression levels of nuclear Nrf2 were markedly increased in the curcumin-treated cells, as compared with in the oxidant-treated cells. Immunocytochemistry detected similar results to the western blot analysis; only minimal positive brown staining was detected in the nuclei of the oxidant-treated cells, as compared with the control cells, in which no positive brown nuclear staining was detected. However, in the curcumin-treated cells, the amount of positive brown staining was abundant in the nucleus, as compared with in the oxidant-treated cells.

These results indicate that oxidative stress may activate the Nrf2 regulatory pathway, and pretreatment with curcumin 
A

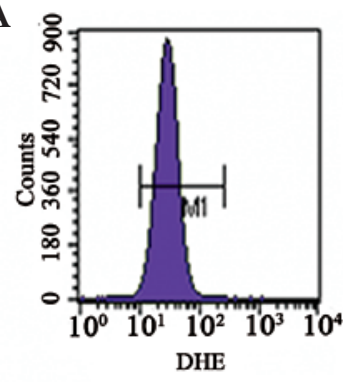

$\mathbf{a}$

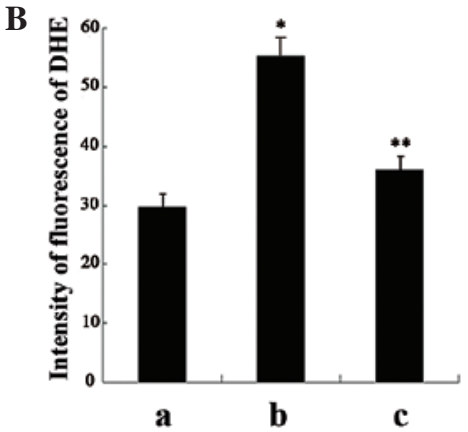

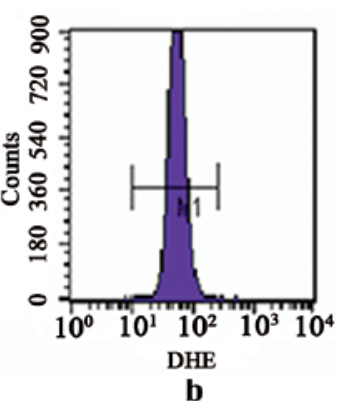

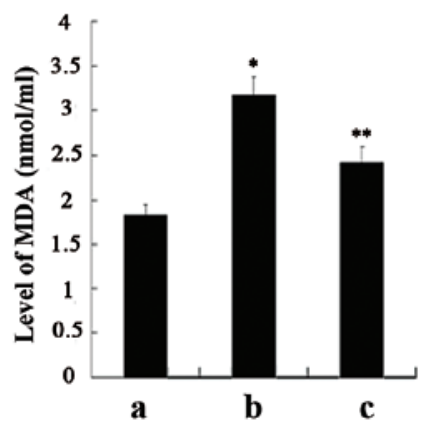

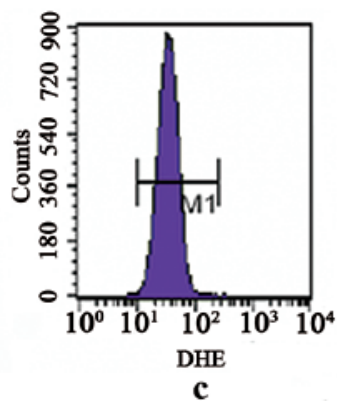

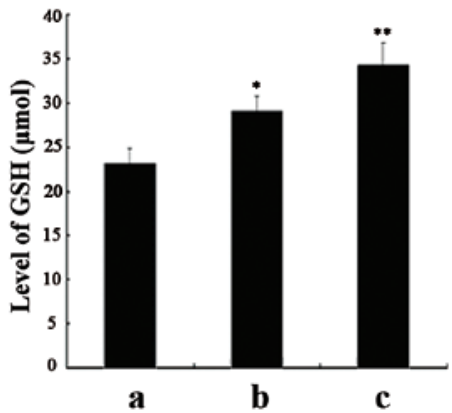

Figure 1. Levels of reactive oxygen species (ROS), as determined using dihydroethidium (DHE); malondialdehyde (MDA); and glutathione (GSH) in various hepatic stellate cell-T6 groups. (A) Analysis of DHE by flow cytometry. (B) Comparison of the levels of ROS, as determined using DHE; MDA; and GSH between the various groups. a, negative control cells; b, oxidant-treated cells; c, curcumin-treated cells. Data are presented as the mean \pm standard deviation. ${ }^{*} \mathrm{P}<0.01$ vs. the negative control cells; ${ }^{* *} \mathrm{P}<0.01$ vs. the oxidant-treated cells.

could protect HSCs against oxidative stress via promoting the translocation of Nrf2 from the cytoplasm to the nucleus.

Curcumin blocks GO-induced $\alpha$-SMA expression and HSC activation. Desmin, which is a type of cytoskeletal intermediate filament, has been widely used as a marker for distinguishing HSCs. Desmin was expressed in all cell groups, as detected using immunofluorescence (Fig. 3A), and there were no differences in desmin expression between the groups, as determined by western blot analysis (Fig. 3B). $\alpha$-SMA is produced by activated HSCs, and is a characteristic signal of HSC activation. As shown in Fig. 3A, no fluorescence expression of $\alpha$-SMA was detected in the negative control cells, whereas following treatment with GO for $2 \mathrm{~h}$ abundant fluorescence expression of $\alpha$-SMA was observed. Furthermore, the fluorescence expression of $\alpha$-SMA in the cells pretreated with curcumin was markedly decreased, as compared with the oxidant-treated cells. Consistent with the alterations in immunofluorescence, the protein expression levels of $\alpha$-SMA were significantly increased in the oxidant-treated cells, as compared with the negative control cells, whereas the expression levels of $\alpha$-SMA were significantly decreased in the curcumin-treated cells, as compared with the oxidant-treated cells. However, the expression levels remained higher in the curcumin-treated cells, as compared with in the negative control cells (Fig. 3B and C).

These results suggest that GO-induced oxidative stress may enhance $\alpha$-SMA expression in HSCs, and the transformation of HSCs to myofibroblast-like cells. Furthermore, treatment with curcumin may activate the Nrf2 regulatory pathway and subsequently suppress $\alpha$-SMA expression and HSC activation, which may be associated with its antifibrotic effects.
Curcumin inhibits the expression of ECM molecules in GO-treated HSCs. Serum levels of PCIII, CIV, LN and HA are often considered indices of liver fibrosis; therefore, the present study aimed to detect the levels of PCIII, CIV, LN and HA in the supernatant (Fig. 4A-D). Compared with the negative control cells, HSCs treated with GO for $2 \mathrm{~h}$ exhibited significantly increased levels of ECM molecules. Conversely, the levels of ECM molecules were markedly reduced following pretreatment with curcumin; however, the levels remained higher, as compared with in the negative control cells.

These results indicate that treatment with GO may markedly increase the expression of ECM molecules in HSCs. Furthermore, the activation of Nrf2 by curcumin may exert inhibitory effects on the expression of ECM molecules in HSC, which may be associated with its antifibrogenic effects.

\section{Discussion}

The Nrf2 pathway is regarded as the most important pathway with regards to cellular protection against oxidative stress (21). As a pivotal modulator of the response to oxidative stress, the activation of Nrf2 induces the expression of various protective antioxidant genes $(22,23)$. Under normal conditions, Nrf2 is sequestered in the cytoplasm where it is bound to the cytoskeleton-associated protein Keap1 (24). Oxidative stress promotes the dissociation of the Nrf2-Keap1 complex, thus resulting in Nrf2 stabilization and translocation to the nucleus (25). In the nucleus, Nrf2 associates with dimerization partners and binds antioxidant-response element sequences, in order to induce the expression of various detoxification and antioxidant enzyme genes that contribute to the protective response (26). 
A
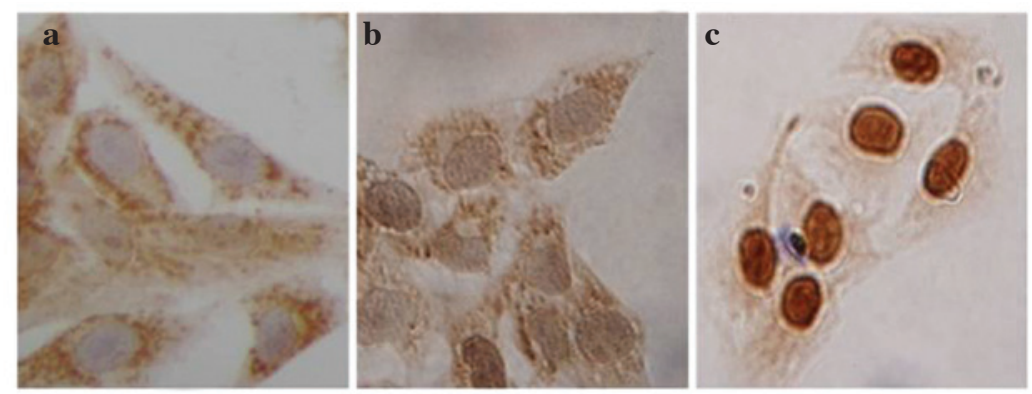

B

Total Nif?

$\beta$-actin

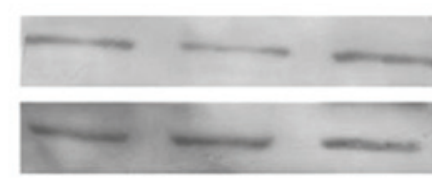

Nuclear Nrf2

$\beta$-actin

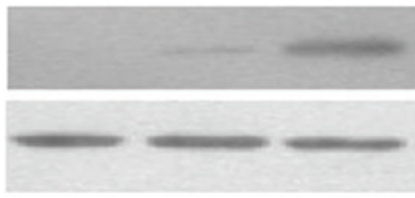

Cytoplasmic Nrf2

$\beta$-actin
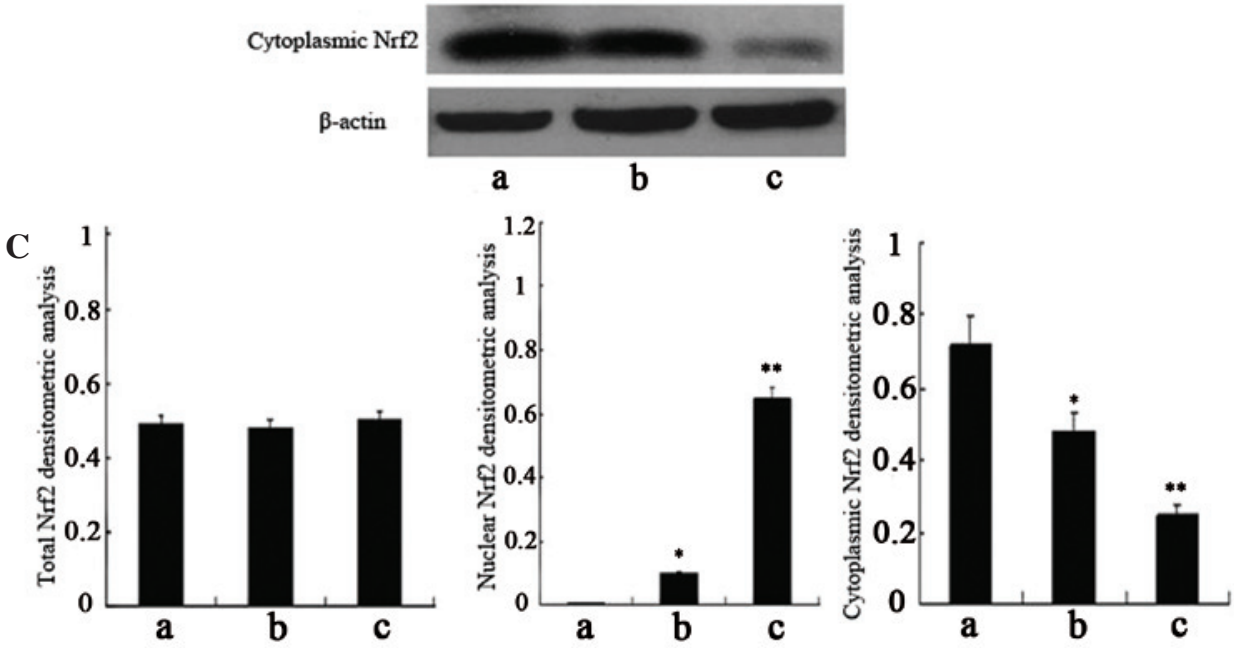

Figure 2. Expression of nuclear factor-erythroid 2-related factor (Nrf2) in various hepatic stellate cell-T6 groups. (A) Expression of Nrf2 in the various groups, as detected by immunocytochemical analysis (original magnification, $\mathrm{x} 400$ ). (B) Expression of Nrf2 in the various groups, as detected by western blot analysis. $\beta$-actin was used as a loading control. (C) Densitometric analysis of western blot results. a, negative control cells; b, oxidant-treated cells; c, curcumin-treated cells. Data are presented as the mean \pm standard deviation. ${ }^{*} \mathrm{P}<0.01$ vs. the negative control cells; ${ }^{* *} \mathrm{P}<0.01$ vs. the oxidant-treated cells.

Curcumin is a natural polyphenol product that is derived from the rhizome of Curcuma longa. Numerous studies have detected the various bioactivities of curcumin, including antioxidant, anti-inflammatory, cell apoptosis-inducing and cell proliferation-inhibiting activities (27-30). In addition, it has been suggested that curcumin may be used clinically against numerous types of cancer, inflammatory bowel disease, irritable bowel syndrome (IBS), rheumatoid arthritis and atherosclerosis (31). Cheng et al (32) detected the effects of curcumin on 25 patients with various types of high-risk or premalignant lesions; following 3 months of treatment with curcumin, some patients exhibited histological improvement in premalignant lesions. Dhillon et al (33) studied the efficacy of curcumin on patients with advanced pancreatic cancer, and demonstrated that oral curcumin administration exerted biological activity in some patients. Hanai et al (34) conducted a double-blind, placebo-controlled trial in 89 patients with ulcerative colitis (UC); curcumin was shown to reduce the incidence of UC and may be considered a promising treatment for UC. In addition, a blind pilot study demonstrated that abdominal pain and the discomfort score of patients with IBS were significantly reduced following treatment with curcumin (35).

Previous studies have detected various functions of curcumin. Notably, curcumin has been shown to function as an exogenous agonist of Nrf2 (19). A previous study suggested that curcumin may alter the conformation of Keap1, and promote the dissociation of Nrf2 from Keap1 and its subsequent nuclear translocation (36). The present study evaluated the effects of curcumin on Nrf2 regulation in HSCs; Nrf2 was localized to the cytoplasm under normal conditions and little Nrf2 was localized to the nucleus following the induction of oxidative stress. However, pretreatment with curcumin induced a substantial localization of Nrf2 to the nucleus in HSCs.

Increased levels of GSH are an index of Nrf2 activation, and increased GSH may be considered a major factor underlying the protection associated with Nrf2 activation $(37,38)$. GSH not 
A
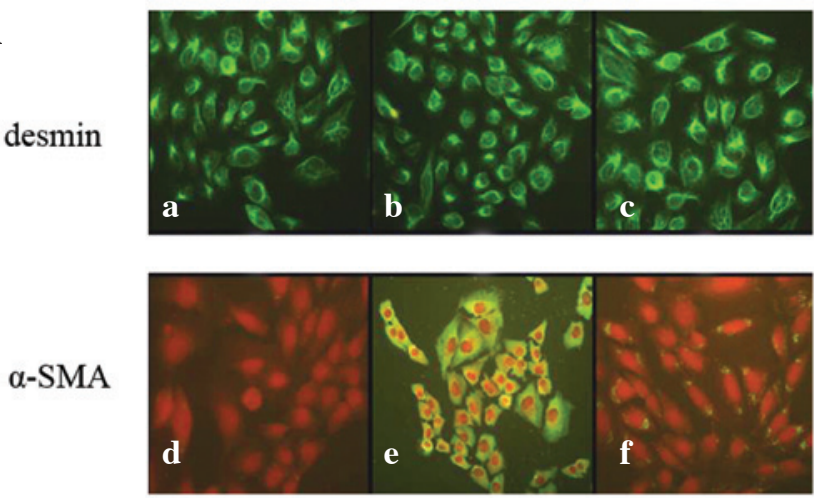

B
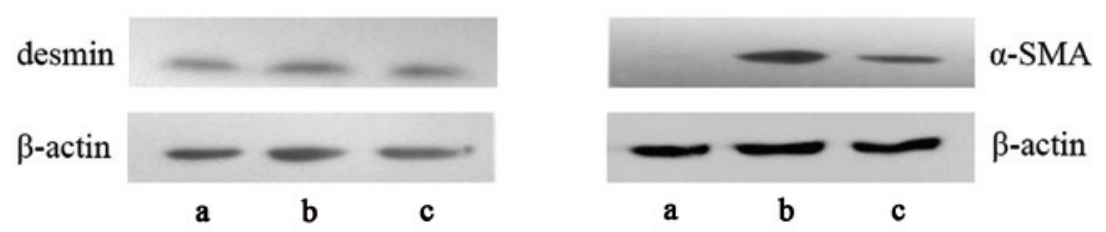

C
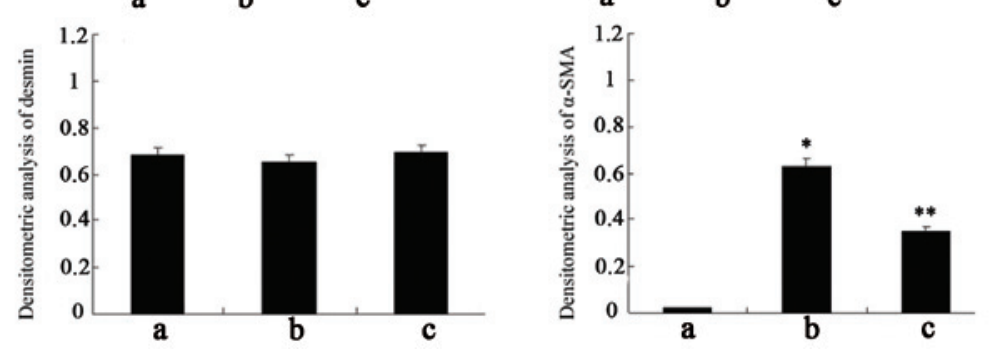

Figure 3. Expression of smooth muscle $\alpha$-actin ( $\alpha$-SMA) and desmin in various hepatic stellate cell (HSC)-T6 groups. (A) Activation of HSC-T6 in the various groups, as determined by immunofluorescence (original magnification, x400). (a-c) Staining assay of desmin in negative control cells, oxidant-treated cells and curcumin-treated cells, respectively; (d-f) staining assay of $\alpha$-SMA in negative control cells, oxidant-treated cells and curcumin-treated cells, respectively. Red staining indicates nuclei, green staining indicates $\alpha$-SMA expression. (B) Expression of desmin and $\alpha$-SMA, as determined by western blot analysis. $\beta$-actin was used as a loading control. (C) Densitometric analysis of western blotting. a, negative control cells; b, oxidant-treated cells; c, curcumin-treated cells. Data are presented as the mean \pm standard deviation. ${ }^{*} \mathrm{P}<0.01$ vs. the negative control cells; ${ }^{* *} \mathrm{P}<0.01$ vs. the oxidant-treated cells.

A

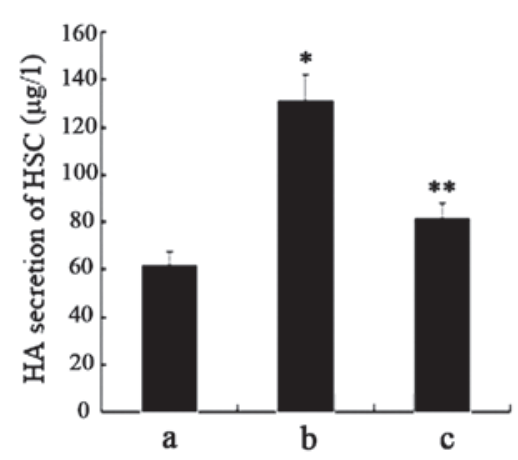

C

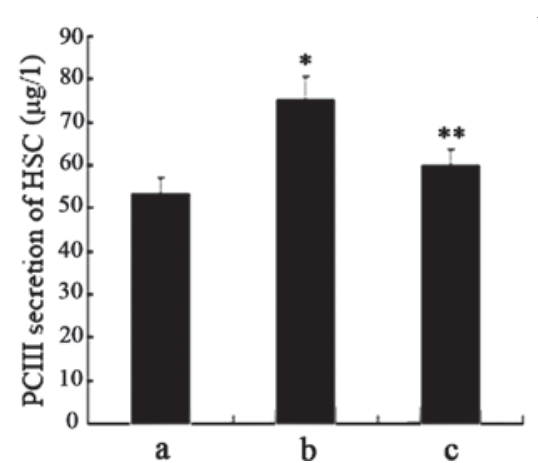

B

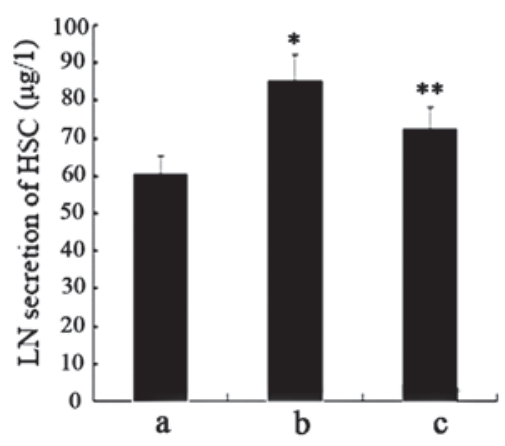

D

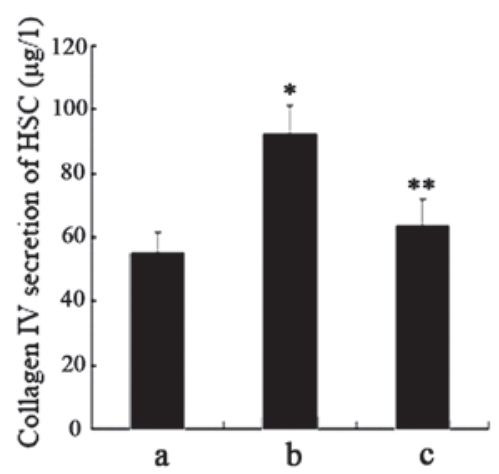

Figure 4. Secretion of components of the extracellular matrix in various hepatic stellate cell-T6 groups. Secretion of (A) hyaluronic acid (HA), (B) laminin (LN), (C) procollagen III (PCIII) and (D) collagen IV from the various cell groups, as determined by radioimmunoassay. a, negative control cells; b, oxidant-treated cells; c, curcumin-treated cells. Data are presented as the mean \pm standard deviation. ${ }^{*} \mathrm{P}<0.01$ vs. the negative control cells; ${ }^{* * *} \mathrm{P}<0.01$ vs. the oxidant-treated cells. 
only produces reducing equivalents, which are necessary for the conversion of $\mathrm{H}_{2} \mathrm{O}_{2}$ and lipid peroxides to water and lipid alcohols (39), but also has an important role in the protection of protein sulfhydration against oxidation (40). The rate-limiting reaction in GSH biosynthesis is catalyzed by GCL, which comprises two subunits: GCLC and GCLM. Nrf2 is able to increase the expression of GCLC and GCLM (41); therefore, the preferential activation of Nrf2 leads to more efficient GSH biosynthesis and improved antioxidant status (42). In the present study, the levels of GSH were increased alongside increasing Nrf2 nuclear translocation following curcumin treatment. GSH elevates the antioxidant ability of cells against oxidative stress.

The pathogenesis of hepatic fibrosis has yet to be completely clarified; however, it is generally accepted that the activation of HSCs is central to the process (43). In the prophase of liver fibrosis, quiescent HSCs transform into myofibroblasts, which are characterized by the assembly of $\alpha$-SMA stress fibers, loss of cytosolic retinol and increased proliferation (44). This activation subsequently results in the synthesis of cytokines and the accumulation of ECM molecules (45). Considerable attention has been focused on elucidating the mechanistic triggers of HSC myofibroblast transformation. In addition, it is hypothesized that oxidative stress may contribute to HSC activation (46).

In the present study, GO reacted with glucose in the culture media resulting in the generation of glucuronic acid and $\mathrm{H}_{2} \mathrm{O}_{2}$. These increased levels of ROS may stimulate HSCs to undergo oxidative stress (47). The results of the present study demonstrated that following treatment with GO, the levels of ROS in the oxidant-treated cells were significantly increased, as compared with in the negative control cells. The negative control cells were quiescent, whereas the oxidant-treated cells were activated by oxidative stress. In addition to the upregulation of Nrf2 nuclear translocation in the curcumin-treated cells, the levels of ROS and HSC activation were decreased, as compared with in the oxidant-treated cells.

In-depth research regarding the mechanisms underlying liver fibrosis has revealed the role of free radicals and membrane lipid peroxidation in the process of liver injury (48). Oxygen radicals attack unsaturated fatty acids in cellular membranes and initiate lipid peroxidation. Lipid peroxidation leads to alterations in the permeability of cellular membranes, subsequently aggravating cell dysfunction and promoting the secretion of ECM molecules $(49,50)$. Not only does oxidative stress stimulate HSC proliferation and collagen synthesis, it also further damages cells by promoting lipid peroxidation. MDA and other peroxidation products increase collagen synthesis via HSC activation, and stimulate Kupffer cells to release cytokines that promote fibrosis (51). Lipid peroxidation has an important role in regulating collagen gene expression, and is associated with cell injury and fibrosis. ROS and lipid peroxidation have been implicated as profibrogenic mediators (52), whereas Nrf2 is effective at suppressing cell damage resulting from lipid peroxidation (53). In the present study, the levels of MDA were increased alongside ROS levels in the oxidant-treated cells; however, following pretreatment with curcumin, the levels of MDA were reduced, as compared with in the oxidant-treated cells.

Liver fibrosis is the excessive deposition of ECM molecules following liver injury. ECM accumulation is associated with increased collagen synthesis and decreased matrix degradation, contributing to liver fibrosis and remodeling (54). Active HSCs are the primary source of the excessive production of ECM components (55), and the ECM enhanced density leads to increased matrix stiffness, which is a significant stimulus for the activation of HSCs (56). In the present study, oxidative stress induced HSC activation, which was followed by the enhanced synthesis of ECM components. Following curcumin pretreatment, the activation of HSCs was inhibited, and the secretion of ECM components was suppressed.

In conclusion, the present study demonstrated that curcumin-induced Nrf2 activation may protect HSCs against oxidative stress-induced injury, and this effect was characterized by enhanced Nrf2 nuclear translocation and antioxidant capacity. The underlying mechanism remains to be elucidated; however, the present study proposes a broader application for $\mathrm{Nrf} 2$ in the prevention and treatment of hepatic damage.

\section{Acknowledgements}

The present study was supported by the National Natural Science Foundation of China (grant nos. 30800515, 81270485 and 81170376) and the Natural Science Foundation of Shaanxi Province (grant no. 2013JM4021).

\section{References}

1. Parola M and Pinzani M: Hepatic wound repair. Fibrogenesis Tissue Repair 2: 4, 2009

2. Loguercio C and Federico A: Oxidative stress in viral and alcoholic hepatitis. Free Radic Biol Med 34: 1-10, 2003.

3. Lin X, Zhang S, Huang R, Wei L, Tan S, Liang S, Tian Y, Wu X, $\mathrm{Lu} \mathrm{Z}$ and Huang Q: Helenalin attenuates alcohol-induced hepatic fibrosis by enhancing ethanol metabolism, inhibiting oxidative stress and suppressing HSC activation. Fitoterapia 95: 203-213, 2014.

4. Lee BH, Hsu WH, Hsu YW and Pan TM: Suppression of dimerumic acid on hepatic fibrosis caused from carboxymethyl-lysine (CML) by attenuating oxidative stress depends on Nrf2 activation in hepatic stellate cells (HSCs). Food Chem Toxicol 62: 413-419, 2013.

5. Bisbal C, Lambert K and Avignion A: Antioxidants and glucose metabolism disorders. Curr Opin Clin Nutr Metab Care 13: 439-446, 2010.

6. Anoush M, Eghbal MA, Fathiazad F, Hamzeiy H and Kouzehkonani NS: The protective effects of garlic extract against acetaminophen-induced oxidative stress and glutathione depletion. Park J Biol Sci 12: 765-771, 2009.

7. Sun Q, Long Z, Wu H, Liu Y, Wang L, Zhang X, Wang X and Hai C: Effect of alcohol on diethylnitrosamine-induced hepatic toxicity: Critical role of ROS, lipid accumulation, and mitochondrial dysfunction. Exp Toxicol Pathol 67: 491-498, 2015.

8. Takemoto K, Hatano E, Iwaisako K, Takeiri M, Noma N, Ohmae S, Toriguchi K, Tanabe K, Tanaka H, Seo S, et al: Necrostatin-1 protects against reactive oxygen species (ROS)-induced hepatotoxicity in acetaminophen-induced acute liver failure. FEBS Open Bio 4: 777-787, 2014.

9. Liu J, Wang X, Liu R, Liu Y, Zhang T, Fu H and Hai C: Oleanolic acid co-administration alleviates ethanol-induced hepatic injury via Nrf-2 and ethanol-metabolizing modulating in rats. Chem Biol Interact 221: 88-98, 2014.

10. Browning JD and Horton JD: Molecular mediators of hepatic steatosis and liver injury. J Clin Invest 114: 147-152, 2004

11. Singh S, Vrishni S, Singh BK, Rahman I and Kakkar P: Nrf2-ARE stress response mechanism: A control point in oxidative stress-mediated dysfunctions and chronic inflammatory diseases. Free Radic Rec 44: 1267-1288, 2010.

12. Matsunami T, Sato Y, Ariga S, Sato T, Kashimura H, Haseqawa Y and Yukawa M: Regulation of oxidative stress and inflammation by hepatic adiponectin receptor 2 in an animal model of nonalcoholic steatohepatitis. Int J Clin Exp Pathol 3: 472-481, 2010. 
13. De Minicis S, Candelaresi C, Agostinelli L, Taffetani S Saccomanno S, Rychlicki C, Trozzi L, Marzioni M, Benedetti A and Svegliati-Baroni G: Endoplasmic Reticulum stress induces hepatic stellate cell apoptosis and contributes to fibrosis resolution. Liver Int 32: 1574-1584, 2012.

14. Tacke $\mathrm{F}$ and Weiskirchen R: Liver fibrosis - pathogenesis and novel therapeutic approaches. Internist (Berl) 51: 21-29, 2010 (In German).

15. Vargas MR and Johnson JA: The Nrf2-ARE cytoprotective pathway in astrocytes. Expert Rev Mol Med 11: e17, 2009.

16. Klaassen CD and Reisman SA: Nrf2 the rescue: Effects of the antioxidative/electrophilic response on the liver. Toxicol Appl Pharmacol 244: 57-65, 2010.

17. Lamlé J, Marhenke S, Borlak J, von Wasielewski R, Eriksson CJ, Geffers R, Manns MP, Yamamoto M and Vogel A: Nuclear factor-eythroid 2-related factor 2 prevents alcohol-induced fulminant liver injury. Gastrornterology 134: 1159-1168, 2008.

18. Bar-Sela G,Epelbaum R and Schaffer M: Curcumin as an anti-cancer agent: Review of the gap between basic and clinical applications. Curr Med Chem 17: 190-197, 2010.

19. Rahman I: Antioxidant therapeutic advances in COPD. Ther Adv Respir Dis 2: 351-374, 2008.

20. Ishiqaki N, Yamamoto N, Jin H, Uchida K, Terai S and Sakaida I: Continuos intravenous infusion of atrial natriuretic peptide (ANP) prevented liver fibrosis in rat. Biochem Biophys Res Commun 378: 354-359, 2009.

21. Copple IM, Goldring CE, Kitteringham NR and Park BK: The Nrf2-Keap1 defense pathway: Role in protection against drug-induced toxicity. Toxicology 246: 24-33, 2008.

22. Mazur W, Lindholm P, Vuorinen K, Myllärniemi M, Salmenkivi K and Kinnula VL: Cell-specific elevation of NRF2 and sulfiredoxin-1 as markers of oxidative stress in the lungs of idiopathic pulmonary fibrosis and non-specific interstitial pneumonia. APMIS 118: 703-712, 2010.

23. Churchman AT, Anwar AA, Li FY, Sato H, Ishii T, Mann GE and Siow RC: Transforming growth factor-beta1 elicits Nrf2-mediated antioxidant responses in aortic smooth muscle cells. J Cell Mol Med 13: 2282-2292, 2009.

24. Chen W, Sun Z, Wang XJ, Jiang T, Huang Z, Fang D and Zhang DD: Direct interaction between Nrf2 and p21 (Cip1/WAF1) upregulates the Nrf2-mediated antioxidant response. Mol Cell 34: 663-673, 2009.

25. Kaspar JW, Niture SK and Jaiswal AK: Nrf2: INrf2 (Keap1) signaling in oxidative stress. Free Radic Biol Med 47: 1304-1309, 2009.

26. Katsuoka F, Motohashi H, Ishii T, Aburatani H, Engel JD and Yamamoto M: Genetic evidence that small maf proteins are essential for the activation of antioxidant response element-dependent genes. Mol Cell Biol 25: 8044-8051, 2005.

27. Ruby AJ, Kuttan G, Babu KD, Rajasekharan KN and Kuttan R: Anti-tumor and antioxidant activity of natural curcuminoids. Cancer Lett 94: 79-83, 1995.

28. Joe B, Rao UJ and Lokesh BR: Presence of acidic glycoprotein in the serum of arthritic rats: Modulation by capsaicin and curcumin. Mol Cell Biochem 169: 125-134, 1997.

29. Xu YX, Pindolina KR, Janakiraman N, Noth C, Chapman RA and Gautam SC: Curcumin, a compound with anti-inflammatory and anti-oxidant properties, down-regulates chemokine expression in bone marrow stromal cell. Exp Hematol 25: 413-422, 1997.

30. Dujic J, Kippenberger S, Ramirez-Bosca A, Diaz-Alperi J, Bereiter-Hahn J, Kaufmann R, Bernd A and Hofmann M Curcumin in combination with visible light inhibits tumor growth in a xenograft tumor model. Int J Cancer 124: 1422-1428, 2009.

31. Fan X, Zhang C, Liu DB, Yan J and Liang HP: The clinical applications of curcumin: Current state and the future. Curr Pharm Des 19: 2011-2031, 2013.

32. Cheng AL, Hsu CH, Lin JK, Hsu MM, Ho YF, Shen TS, Ko JY, Lin JT, Lin BR, Ming-Shiang W, et al: Phase I clinical trial of curcumin, a chemopreventive agent, in patients with high-risk or pre-malignant lesions. Anticancer Res 21: 2895-2900, 2001.

33. Dhillon N, Aggarwal BB, Newman RA, Wolff RA, Kunnumakkara AB, Abbruzzese JL, Ng CS, Badmaev V and Kurzrock R: Phase II trial of curcumin in patients with advanced pancreatic cancer. Clin Cancer Res 14: 4491-4499, 2008.

34. Hanai H, Iida T, Takeuchi K, Watanabe F, Maruyama Y, Andoh A, Tsujikawa T, Fujiyama Y, Mitsuyama K, Sata M, et al: Curcumin maintenance therapy for ulcerative colitis: Randomized, multicenter, double-blind, placebo-controlled trial. Clin Gastroenterol Hepatol 4: 1502-1506, 2006.
35. Bundy R, Walker AF, Middleton RW and Booth J: Turmeric extract may improve irritable bowel syndrome symptomology in otherwise healthy adults: A pilot study. J Altern Complement Med 10: 1015-1018, 2004.

36. Balogun E, Hoque M, Gong P, Killeen E, Green CJ, Foresti R, Alam J and Motterlini R: Curcumin activates the haem oxygenase-1 gene via regulation of Nrf2 and the antioxidant-responsive element. Biochem J 371: 887-895, 2003.

37. Ma ZC, Hong Q, Wang YG, Tan HL, Xiao CR, Liang QD, Zhang BL and Gao Y: Ferulic acid protects human umbilical vein endothelial cells from radiation induced oxidative stress by phosphatidylinositol 3-kinase and extracelluar signal-regulated kinase pathways. Biol Pharm Bull 33: 29-34, 2010.

38. Stridh MH, Correa F, Nodin C, Weber SG, Blomstrand F, Nilsson M and Sandberg M: Enhanced glutathione efflux from astrocytes in culture by low extracellular $\mathrm{Ca}^{2+}$ and curcumin. Neurochem Res 35: 1231-1238, 2010.

39. Ballatori N, Krance SM, Notenboom S, Shi S, Tieu K and Hammond CL: Glutathione dysregulation and the etiology and progression of human disease. Biol Chem 390: 191-214, 2009.

40. Bindoli A, Fukuto JM and Forman HJ: Thiol chemistry in peroxidase catalysis and redox signaling. Antioxid Redox Signal 10: 1549-1564, 2008

41. Johnson JA, Johnson DA, Kraft AD, Calkins MJ, Jakel RJ, Vargas MR and Chen PC: The Nrf2-ARE pathway: An indicator and modulator of oxidative stress in neurodegeneration. Ann N Y Acad Sci 1147: 61-69, 2008.

42. Kraft AD, Johnson DA and Johnson JA: Nuclear factor E2-related factor 2-dependent antioxidant response element activation by tert-butylhydroquinone and sulforaphane occurring preferentially in astrocytes conditions neurons against oxidative insult. J Neurosci 24: 1101-1112, 2004.

43. ReichardJFand PetersenDR:Hepatic stellatecells lack AP-1 responsiveness to electrophiles and phorbol 12-myristate-13-acetate. Biochem Biophys Res Commun 322: 842-853, 2004.

44. Maeda K, Koda M, Matono T, Sugihara T, Yamamoto S, Ueki M, Murawaki Y, Yamashita N and Nishiyama S: Preventive effects of ME3738 on hepatic fibrosis induced by bile duct ligation in rats. Hepatol Res 38: 727-735, 2008.

45. Urtasun R, Conde de la Rosa L and Nieto N: Oxidative and nitrosative stress and fibrogenic response. Clin Liver Dis 12: 769-790, 2008.

46. Wang J, Leclercq I, Brymora JM, Xu N, Ramezani-Moghadam M, London RM, Brigstock D and George J: Kupffer cells mediate leptin-induced liver fibrosis. Gastroenterology 137: 713-723, 2009.

47. Beyer TA, Xu W, Teupser D, auf dem Keller U, Bugnon P, Hildt E, Thiery J, Kan YW and Werner S: Impaired liver regeneration in Nrf2 knockout mice: Role of ROS-mediated insulin/IGF-1 resistance. EMBO J 27: 212-223, 2008.

48. Qin Y and Tian YP: Preventive effects of chronic exogenous growth hormone levels on diet-induced hepatic steatosis in rats. Lipids Health Dis 9: 78, 2010.

49. Kulbacka J, Saczko J and Chwiłkowska A: Oxidative stress in cells damage processes. Pol Merkur Lekarski 27: 44-47, 2009 (In Polish).

50. Moselhy SS and Ali HK: Hepatoprotective effect of cinnamon extracts against carbon tetrachloride induced oxidative stress and liver injury in rats. Biol Res 42: 93-98, 2009.

51. Sener G, Kabasakal L, Yüksel M, Gedik N and Alican Y: Hepatic fibrosis in biliary-obstructed rats is prevented by Ginkgo biloba treatment. World J Gastroenterol 11: 5444-5449, 2005 .

52. Kim J, Seok YM, Jung KJ and Park KM: Reactive oxygen species/oxidative stress contributes to progression of kidney fibrosis following transient ischemic injury in mice. Am J Physiol Renal Physoil 297: F461-F470, 2009.

53. Osburn WO, Wakabayashi N, Misra V, Nilles T, Biswal S, Trush MA and Kensler TW: Nrf2 regulates an adaptive response protecting against oxidative damage following diquat-mediated formation of superoxide anion. Arch Biochem Biophys 454: $7-15,2006$.

54. Snyder JC, Zemke AC and Stripp BR: Reparative capacity of airway epithelium impacts deposition and remodeling of extracellular matrix. Am J Respir Cell Mol Biol 40: 633-642, 2009.

55. Friedman SL: Mechanisms of hepatic fibrogenesis. Gastroenterology 134: 1655-1669, 2008.

56. Wells RG: The role of matrix stiffness in regulating cell behavior. Hepatology 47: 1394-1400, 2008. 